\title{
Usuários de álcool e outras drogas na Rede de Atenção Psicossocial: Cuidado de Enfermagem
}

\section{Users of Alcohol and other Drugs in the Psychosocial Care Network: Nursing Care}

\author{
Usuarios de alcohol y otras drogas en la Red de Atención Psicosocial:
}

\section{Atención de Enfermería}

\author{
Priscilla Mecia Conceição de Brito ${ }^{1}$, ORCID 0000-0003-1477-0674 \\ Manuela Bastos Alves ${ }^{2}$, ORCID 0000-0002-4073-5146 \\ Verônica Mascarenhas Oliveira ${ }^{3}$, ORCID 0000-0002-4283-9897 \\ Maria do Perpetuo Socorro de Sousa Nóbrega ${ }^{4}$, ORCID 0000-0002-4974-0611 \\ Simone Conceição Oliveira Baptista ${ }^{5}$, ORCID 0000-0002-5266-4912 \\ Rudval Souza da Silva ${ }^{6}$ ORCID 0000-0002-7991-8804 \\ 1236 Universidade do Estado da Bahia, Brasil \\ ${ }^{4}$ Universidade de São Paulo, Brasil \\ ${ }^{5}$ Universidade Federal da Bahia, Brasil
}

\begin{abstract}
Resumo: Objetivo: Analisar a produção do cuidado da equipe de enfermagem aos usuários de álcool e outras drogas na Rede de Atenção Psicossocial. Método: Estudo exploratório e descritivo de abordagem qualitativa, desenvolvido com 11 profissionais da equipe de enfermagem atuantes em três serviços de saúde da Rede, localizados num município do interior da Bahia, Brasil. Os dados foram coletados entre dezembro de 2017 e fevereiro de 2018 utilizando-se da entrevista semiestruturada. Resultados: Os resultados apontam para um acesso dos usuários a Rede a partir de encaminhamentos e referências; as atividades de acolhimento têm sido desenvolvidas apenas pelos Centros de Atenção Psicossocial e o vínculo tem se demonstrado como frágil. Conclusão: Conclui-se que o cuidado de enfermagem na Rede tem se desenvolvido de modo fragmentado e pontual, logo, dispositivos como acesso, acolhimento e vínculo devem ser trabalhados com toda equipe de saúde em prol de uma assistência integral.
\end{abstract}

Palavras-chave: serviços de saúde mental; atenção à saúde; acesso aos serviços de saúde; acolhimento; enfermagem.

Abstract: Objective: To analyse the production of care provided by the nursing team to users of alcohol and other drugs in the Psychosocial Care Network. Methods: Exploratory and descriptive study with a qualitative approach, developed with 11 professionals from the 
nursing team working in three health services of the Network, located in a city in the interior of Bahia, Brazil. Data were collected between December 2017 and February 2018 using semi-structured interviews. Results: The results point to users' access to the Network based on refer and reference; the reception activities have been developed only by the Psychosocial Care Centres and the emotional bond has been shown to be fragile. Conclusion: It is concluded that nursing care in the Network has been developed in a fragmented and punctual way, therefore, devices such as access, reception and bonding must be worked with the entire health team in favour of comprehensive care.

Keywords: mental health services; health care; access to health services; reception; nursing.

Resumen: Objetivo: Analizar la producción de cuidados del equipo de enfermería para usuarios de alcohol y drogas en la Red de Atención Psicosocial. Método: Estudio exploratorio y descriptivo con enfoque cualitativo, desarrollado con 11 profesionales del equipo de enfermería que trabajan en tres servicios de salud de la Red, ubicados en una ciudad del interior de Bahía, Brasil. Los datos fueron recolectados entre diciembre de 2017 y febrero de 2018 mediante entrevista semiestructurada. Resultados: Los resultados apuntan al acceso de los usuarios a la Red a través de reenvío y referencia; las actividades de acogida han sido desarrolladas únicamente por los Centros de Atención Psicosocial y se ha demostrado que el vínculo es frágil. Conclusión: Se concluye que el cuidado de enfermería en la Red se ha desarrollado de manera fragmentada y puntual, por lo que dispositivos como el acceso, la recepción y el vínculo deben trabajarse con todo el equipo de salud a favor de la atención integral.

Palabras claves: servicios de salud mental; cuidados de la salud; acceso a servicios de salud; recepción; enfermería.

Recebido: 17/04/2021

Aceito: 29/10/2021

Como citar:

De Brito PMC, Alves MB, Oliveira VM, Nóbrega MdPSdS, Baptista SCO, Da Silva RS. Usuários de álcool e outras drogas na Rede de Atenção Psicossocial: Cuidado de Enfermagem. Enfermería: Cuidados Humanizados. 2021;10(2):175-190. DOI: 10.22235/ech.v10i2.2546

Correspondência: Rudval Souza da Silva. E-mail: rudvalsousa@yahoo.com.br

\section{Introdução}

A questão do consumo de álcool e outras drogas têm se caracterizado como um dos principais problemas de saúde pública no mundo, segundo relatório da Organização Mundial de Saúde. Dados apontam que 4,8 \% das pessoas independente de sexo, idade, raça ou nível de escolaridade já fizeram uso de algum tipo de droga ilícita. ${ }^{(1)}$ Informações divulgadas no Relatório Mundial sobre Drogas, publicado em 2018, revelam uma expansão do mercado mundial de substâncias psicoativas, ilícitas, tendo o ópio, a cocaína e a cannabis, ocupando lugar de destaque dentre as drogas mais consumidas em 2016, incluindo o cenário brasileiro. ${ }^{(2)}$ 
De acordo com o $3 .^{\circ}$ Levantamento Nacional sobre uso de drogas pela população brasileira, 3,2 \% dos indivíduos fizeram uso de substâncias ilícitas nos 12 meses anteriores à pesquisa, o que corresponde a 4,9 milhões de pessoas. Neste cenário, vale ressaltar que grande parte dos dados mais alarmantes estão relacionados ao uso abusivo de álcool, o que se configura como um problema de saúde pública demandando a reformulação das práticas de cuidados direcionadas a esses usuários com foco na saúde mental. ${ }^{(3)}$

Historicamente, por um longo tempo, o modelo de atenção à saúde mental esteve centrado apenas na doença, numa perspectiva hospitalocêntrica, constituindo-se como um campo de exclusão. Discussões acerca da cronificação dos usuários, do modelo biomédico, da violação dos direitos humanos e de cidadania fizeram emergir iniciativas políticas, científicas e sociais trazendo uma nova forma de pensar sobre o processo saúde-doença pautado na valorização do cuidado ao indivíduo. ${ }^{(4)}$

Nesta perspectiva surgiu a Rede de Atenção Psicossocial (RAPS), instituída pela Portaria $n^{\circ}$ 3.088/2011 do Ministério de Saúde do Brasil, como uma estratégia de enfrentamento das vulnerabilidades, agravos e doenças que acometem a população. A Rede tem como proposta a reorganização dos serviços de saúde mental de forma integrada, diversificando e ampliando as ações e os equipamentos de saúde, visando garantir o acesso universal e o cuidado integral para as pessoas em sofrimento psíquico. ${ }^{(5)}$

O universo de atuação da RAPS é formado diretamente pelos serviços da atenção básica, com a Estratégia de Saúde da Família, as Unidades Básicas de Saúde e os consultórios de rua; os serviços de atenção hospitalar, com leitos psiquiátricos nos hospitais gerais, urgência e emergência; serviços especializados, a exemplo dos Centros de Atenção Psicossocial (CAPS); além dos serviços de residências terapêuticas. ${ }^{(6)}$

Sobre os serviços especializados, vale destaque para as modalidades dos CAPS: adulto, infantil e álcool e drogas, cuja finalidade centra-se no desempenho de papel estratégico na articulação da rede no território, significando um avanço importante na atenção à saúde mental, a partir de um modelo aberto e de base comunitária, garantindo a livre circulação das pessoas pelos serviços de saúde e demais locais da comunidade. ${ }^{(7)}$

Nessa perspectiva, alinhado ao princípio do cuidado integral, reconhece-se que o problema da drogadição é de caráter multifatorial e necessita de uma diversidade de intervenções a exemplo da ampliação da cobertura e da participativa atuação dos profissionais da saúde de forma a atender as necessidades individuais dos usuários, dando enfoque ao prejuízo trazido pelo uso indevido de álcool e outras drogas, a reabilitação psicossocial e a política de redução de danos. ${ }^{(8-9)}$

Nesse cenário, a Enfermagem Psiquiátrica no âmbito nacional e internacional é reconhecida como especialidade profissional significativa, por melhorar o acesso e a qualidade nos serviços da rede de saúde mental como parte das demandas de saúde pública, possuindo boa capacidade de inserção em equipes multidisciplinares. Os profissionais da equipe de enfermagem se constituem num pilar fundamental para o cuidado dos usuários da rede, uma vez que em todos os dispositivos que compõem a RAPS, esta equipe se encontra presente. ${ }^{(9)}$

A equipe de enfermagem numa perspectiva de atuação interdisciplinar tem o compromisso e responsabilidade em desenvolver um modelo de atenção psicossocial que seja capaz de estabelecer relações participativas com os usuários, assim como construir propostas terapêuticas que estejam comprometidas com as diretrizes da reforma psiquiátrica brasileira, buscando exercer um cuidado humanizado que reconheça o sujeito como protagonista e considere sua individualidade com foco na multidimensionalidade como um ser biopsicossócioespiritual. ${ }^{(5,8-9)}$ 
Assim, o cuidado profissional de enfermagem para usuários de álcool e outras drogas na RAPS deve ser pautado numa perspectiva da clínica ampliada onde os profissionais possam romper barreiras com a hospitalização e o isolamento, consolidando uma atenção que compreenda ações intersetoriais e interdisciplinares capazes de favorecer o acompanhamento contínuo e a vinculação dos usuários aos serviços que compõem a atenção estratégica, básica e hospitalar. ${ }^{(5)}$

Para a compreensão do que é a clínica ampliada é importante retomar o conceito de produção do cuidado como um novo paradigma que emerge como proposição para romper com a tradicional prática assistencial centrada no saber médico e numa clínica baseada exclusivamente na dimensão biológica. A produção do cuidado assume como cerne do processo, o usuário e aqui vale destacar que no contexto da saúde mental, esse é representado pelos usuários de álcool e outras drogas e suas famílias. Assim, o cuidado é produzido a partir de momentos coletivos entre usuários, família e trabalhadores da saúde. ${ }^{(10)}$

Nesse estudo, propõe-se analisar a interrelação entre usuários de álcool e outras drogas, acompanhados na RAPS, e os trabalhadores da equipe de enfermagem, para assim identificar e compreender como ocorre a produção do cuidado nesses cenários.

Assim, este estudo tem como objetivo analisar a produção do cuidado da equipe de enfermagem aos usuários de álcool e outras drogas na Rede de Atenção Psicossocial.

\section{Metodologia}

Trata-se de uma pesquisa exploratória e descritiva, de abordagem qualitativa que se ancora nas peculiaridades relacionas ao paradigma construtivista ${ }^{(11)}$ e as bases da pesquisa social, ${ }^{(12)}$ considerando sua aproximação com a temática da produção do cuidado na RAPS enquanto um fenômeno social que assume uma complexidade inerente a atenção psicossocial. A pesquisa atendeu aos passos recomendados pelos Critérios Consolidados para Relatar uma Pesquisa Qualitativa (COREQ). ${ }^{(13)}$

O estudo foi realizado em três unidades de saúde, a saber: Centro de Atenção Psicossocial na especialidade álcool e drogas (CAPS AD); Centro de Atenção Psicossocial modalidade I (CAPS I) e em uma equipe de Estratégia de Saúde da Família (ESF), todas localizadas em um município do interior da Bahia - Brasil.

Tais unidades integram a Rede de Atenção à Saúde do Município onde ocorreu o estudo, sendo a unidade de Estratégia de Saúde da Família (ESF) um serviço complementar do modelo assistencial da Atenção Básica, que se fundamenta no trabalho em equipe multiprofissional no território. As outras duas unidades (CAPS I e CAPS AD), são unidades especializadas em saúde mental, compondo a RAPS e realizam acompanhamento, tratamento e reinserção social de pessoas com transtorno mental grave e persistente, atuando também numa perspectiva de atendimento interdisciplinar, contando de uma equipe multiprofissional, na qual está inserida a equipe de enfermagem.

O presente estudo é parte integrante de uma Pesquisa de Mestrado desenvolvida no Programa de Pós-graduação em Saúde Coletiva da Universidade Estadual de Feira de Santana (UEFS).

Participaram do estudo seis enfermeiras e cinco técnicas de enfermagem, integrantes das equipes dos serviços supramencionados, que responderam a uma entrevista semiestruturada guiada pela questão norteadora "Como são produzidos os cuidados de enfermagem com os usuários de álcool e outras drogas acompanhados na RAPS?”. O instrumento contemplou questões relacionadas a caracterização sociodemográficas e laboral 
das participantes e foi validado por pesquisadores do grupo de pesquisa com experiência na área temática.

Foi assumido como critério de inclusão a atuação no serviço há mais de seis meses e de exclusão aqueles servidores afastados por férias, licença ou assumindo cargo de gestão. Todas as participantes aceitaram voluntariamente colaborar com o estudo e assinaram o Termo de Consentimento Livre e Esclarecido.

Das 13 profissionais das equipes de enfermagem atuantes nos três cenários e que atendiam aos critérios de inclusão, apenas duas técnicas de enfermagem se recusaram a participar do estudo.

A coleta de dados ocorreu entre dezembro de 2017 e fevereiro de 2018, por entrevistas realizadas em local reservado, com duração média de 30 minutos, utilizando-se do gravador de voz. Em seguida, as falas foram transcritas e organizadas para análise conforme a Técnica de Análise de Conteúdo de Bardin (14).

A Análise de Conteúdo Temática (14) seguiu três etapas: 1) a pré-análise, na qual foram organizados os dados do material empírico; realizada leitura flutuante das falas dos entrevistados e conduzindo para a identificação das unidades de registro; 2) a exploração do material permitiu aos pesquisadores proceder com os recortes das falas dos entrevistados transformando-as em unidades de registros, as quais foram classificadas e codificadas e por fim agrupadas em unidades de significados com base nas convergências e divergências, emergindo assim as categorias temáticas; 3) o tratamento dos resultados conduziu para o momento de priorizar as categorias de maior relevância com base no objeto de estudo para em seguida realizar a interpretação e discussão, de acordo com o quadro teórico e com base na literatura.

Para garantir o anonimato, as participantes foram identificadas com códigos com base na modalidade do serviço (ESF para Estratégia de Saúde da Família; CAPS I para Centro de Atenção Psicossocial I e CAPS AD para CAPS Álcool e Drogas).

O projeto e a condução da pesquisa respeitaram todas as recomendações e preceitos éticos conforme determina a Resolução no 466/12 do Conselho Nacional de Saúde e foi aprovado pelo Comitê de Ética em Pesquisa sob parecer ${ }^{\circ} 2.420 .638$.

\section{Resultados}

Participaram do estudo 11 profissionais da equipe de enfermagem atuantes nos três serviços da RAPS, sendo nove deles do sexo feminino e dois masculinos; na sua maioria, oito delas com idade superior a 30 anos e atuando no serviço com um tempo que variou entre seis meses e 24 anos, sendo nove delas atuavam há mais de dois anos no serviço.

A análise dos dados possibilitou emergir três categorias temáticas que buscam representar como se dá a produção do cuidado pela equipe de enfermagem na RAPS, a saber: 1) Acesso aos serviços a partir da RAPS; 2) Acolhimento dos usuários na RAPS e 3) Vínculo entre a equipe de enfermagem e usuários na RAPS, que caracterizam o cuidado produzido pelos profissionais, conforme apresentadas na Figura 1. 


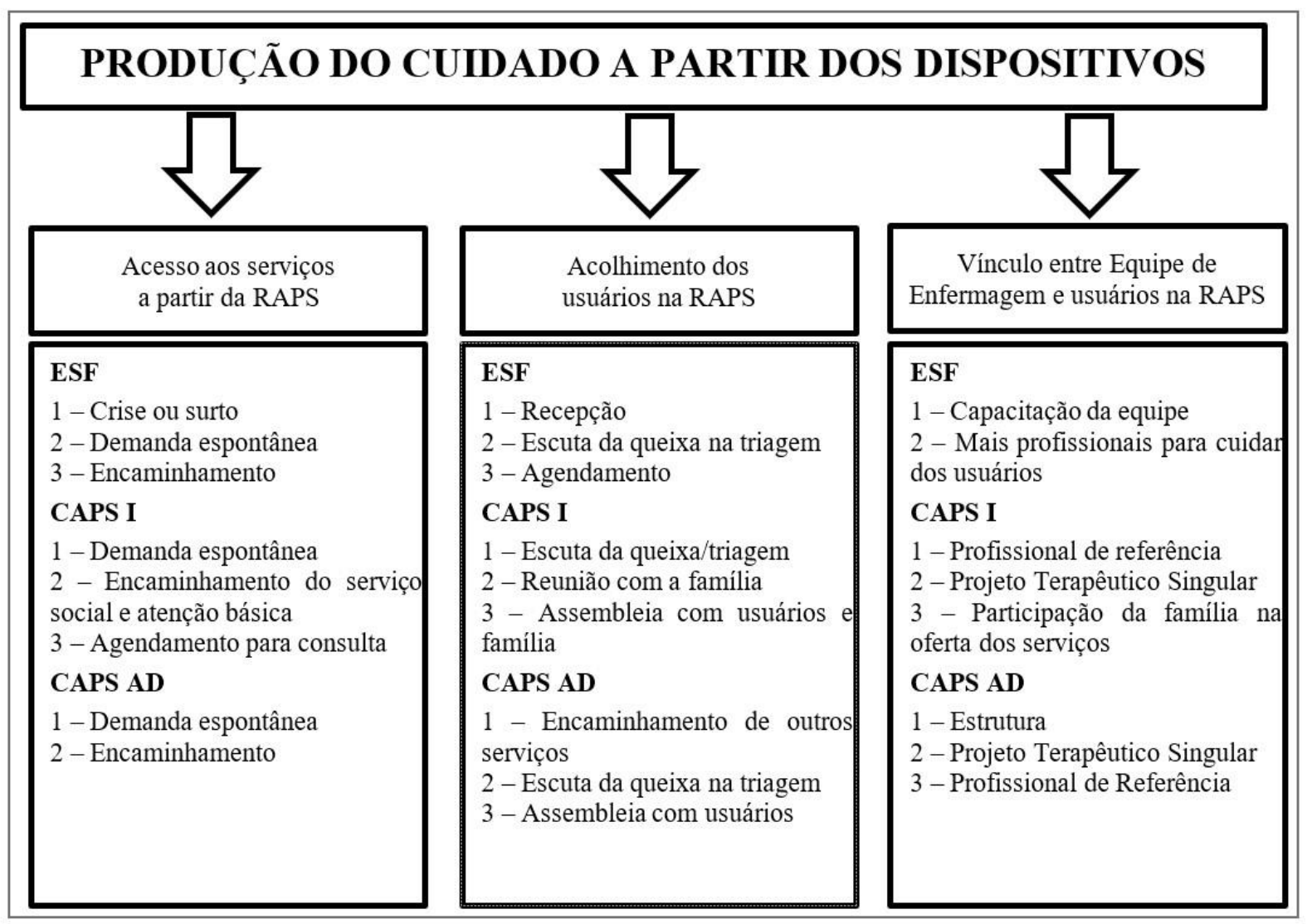

Figura 1. Produção do cuidado e os dispositivos da RAPS. Bahia, Brasil, 2018. Fonte: Criação do autor (2021).

A figura representa as três categorias temáticas a partir dos dispositivos de produção do cuidado (acesso, acolhimento e vínculo) na perspectiva das trabalhadoras do campo da enfermagem nos três cenários que constituem a RAPS e o percurso dos usuários, com transtorno mental grave e persistente, em busca por um cuidado manifestado pelo acompanhamento, tratamento ou reinserção social.

A seguir apresentam-se as categorias com respectivas definições e unidades de registros com base no como é produzido o cuidado aos usuários com transtorno mental, na perspectiva da equipe de enfermagem.

\section{Categoria 1: Acesso aos serviços da RAPS}

Essa categoria contempla a compreensão da equipe de enfermagem sobre como os usuários de álcool e outras drogas acessam os serviços da RAPS, em busca de cuidado. Nas unidades de registros apresentadas a seguir, é possível observar que os participantes apontam que o acesso acontece por demanda espontânea ou encaminhamentos feitos pela equipe da ESF ou Centro de Referência de Assistência Social (CREAS). 
Eles têm acesso aqui quando estão em crise, é muito raro agendar consulta, encaminhamos para o CAPS, que lá é que faz acompanhamento destes pacientes $(E S F)$.

Vem referenciado do CREAS e da ESF, ou demanda espontânea, aí a gente busca as parcerias do CAPS AD (CAPS I).

Se ocorrer uma demanda espontânea ou encaminhada, o usuário será acolhido e atendido (CAPS AD).

Muitas vezes o acesso se dá a partir do agendamento da consulta que é realizada por profissionais de cada serviço ao qual o usuário está vinculado, como segue:

Em alguns casos de necessidade de urgência, de pressão elevada, uma temperatura alta, tudo isso a gente tria e é agendado aqui mesmo (ESF).

Os agendamentos com o psiquiatra são feitos terças e quartas-feiras no período da manhã, triagem é feita segunda, terça e quarta à tarde importante lembrar que estes atendimentos são para nossa demanda, não é para os usuários ambulatoriais (CAPS I).

A gente trabalha com agendamento, se chegar aqui e a família diz que ele não está bem, que a medicação não está fazendo aquele efeito esperado, ele é atendido (CAPS AD).

Os casos de crises, condição característica que fazem os usuários buscarem a RAPS, acontecem de forma pontual, como pode ser observado nas unidades de registro destacadas a seguir:

Quando chegam aqui em crise são medicados e posteriormente liberados e orientados a procurar os CAPS (ESF).

Se não são pacientes nosso, prestamos o primeiro atendimento, medicamos $e$ solicitamos o SAMU para levar para o hospital (CAPS I).

\section{Categoria 2 - Acolhimento dos usuários nos serviços da RAPS}

$\mathrm{O}$ acolhimento, para as participantes do estudo que não atuam no CAPS AD, se configura a partir de um fluxo unidirecional e pautado por uma demanda espontânea. As unidades de registros trazer a descrição da compreensão dessas profissionais quanto ao cuidado na condução do acolhimento aos usuários de álcool e outras drogas.

Quando eles precisam deste atendimento vai ser direcionado para recepção onde será feita a escuta e depois para triagem e, para o atendimento (ESF).

A gente escuta e procura saber o que ele deseja, se está precisando de algo, a necessidade dele do momento, na triagem, e depois a gente encaminha (CAPS I).

Primeiro contato é na recepção, faz a ficha, eu acolho e depois eu referencio (ESF). 
Diferentemente do que acontece na fala dos trabalhadores do CAPS AD, que conduz um acolhimento, escuta ativa e ações interdisciplinares:

Ele chega, a recepção acolhe e encaminha para mim (enfermeiro), eu acolho, escuto o problema dele e trio e encaminho para médica, se precisar ele ser atendido no mesmo dia eu encaminho para ela, senão a gente marca uma consulta (CAPS AD).

O paciente vai chegar, ter o acolhimento, a gente vai sentar-se com ele e com a família e vai escutar o que levou ele procurar o serviço (CAPS AD).

Observa-se que as estratégias utilizadas para o acolhimento aos usuários no contexto da ESF, não apresentam contribuições para o acolhimento do usuário numa perspectiva integradora e direcionada pelas diretrizes da política de saúde mental e da própria RAPS. Já para as profissionais do CAPS AD, apesar de reconhecerem a possibilidade de estratégias para o acolhimento, como reuniões com família e assembleias, observa-se que estas não são postas em prática.

Não tem não, como já tiveram grupos dos programas antes, essas atividades devem serfeitas no CAPS aqui não tem não (ESF).

Reunião com a família que acontece uma vez semana e a assembleia que acontece a cada 15 dias com os usuários e com a família, mas não está tendo nessa frequência (CAPS I).

Nas reuniões e assembleias a gente escuta também a família, posteriormente se senta com a equipe para melhor cuidar deles, mas, no momento não tem ocorrido [reuniões familiares] (CAPS AD).

\section{Categoria 3 - Vínculo entre a equipe de enfermagem e usuários da RAPS}

Esta categoria aponta para as principais características quanto a produção de vínculo entre a equipe de enfermagem e os usuários de álcool e outras drogas na RAPS, considerando que é a partir desse vínculo que a produção do cuidado se torna possível.

É relação de confiança entre nós e os usuário, ficam à vontade quando confiam $(E S F)$.

Estar mais próximo deles, criar laços de afeto, atenção, cuidado (CAPS I).

Ouvir de forma atenta e buscar entender sua necessidade para que ele confie sempre mais em você e [profissional] dá atenção (CAPS AD).

Para tanto, o vínculo pode se dá a partir de um profissional de referência da equipe com o qual o usuário melhor se identifique e o estabeleça.

Todos são referência, mas sempre tem um específico (CAPS I).

Todo mundo é referência de alguém, aquele paciente que tem mais aproximação então a gente vai procurar o profissional que o paciente se abriu mais (CAPS AD).

Entretanto, para alguns participantes desse estudo, o enfermeiro do serviço, é considerado o profissional de referência, caracterizando uma transferência de 
responsabilidade e até negando a possibilidade de outros integrantes da equipe assumirem o papel de ser um profissional de referência, como pode ser observado nas unidades de registro apresentadas a seguir:

Aqui no posto não (tem o profissional de referência), tem no CAPS o psiquiatra, enfermeiro, psicólogo, toda uma equipe (ESF).

Sim, o profissional de referência no caso são os enfermeiros, principalmente, a coordenadora (CAPS I).

Não existe profissional de referência, somos poucos aqui, não tem como (ESF).

No contexto do estabelecimento de vínculo, ao serem questionados sobre a forma de como se estabelece tal construção, o desenvolvimento do Projeto Terapêutico Singular(PTS) tem sido a estratégia mais utilizada pelos trabalhadores dos CAPS.

A equipe conversa e avalia um plano terapêutico conforme necessidade de cada um, mas não conseguimos fazer ainda (CAPS AD).

Com a família, com o usuário e com o profissional, a gente junta os profissionais enfermeiro, psicólogo, assistente social e marca um dia e a família vem com o usuário e em cima do diagnóstico dele a gente constrói o projeto terapêutico (CAPS I).

Após receber o usuário, são feitos o acolhimento e a triagem depois os profissionais sentam e ai é feito um plano terapêutico (CAPS AD).

Essa estratégia é desconhecida por aqueles que atuam para além do CAPS, ao afirmar desconhecer o que é o PTS, como nas unidades de registro dos trabalhadores da ESF:

Projeto terapêutico? Não sei, acho que quem te poderia dizer é a coordenadora. Desconheço (ESF).

Não temos, não sei como é feito (ESF).

Não trabalhamos com PTS, não tem como, deve ser feito nos CAPS (ESF).

\section{Discussão}

A partir do movimento da reforma psiquiátrica brasileira iniciado na década de 1960, tem-se buscado estratégias para produção do cuidado à pessoa com transtorno mental que extrapole a dimensão asilar e opressora, repensando o cuidado como um movimento dinâmico que envolve o ato de ensinar, o encontro com o outro e o respeito à livre produção subjetiva da realidade social de cada ser ${ }^{(15)}$ de modo que as particularidades sejam levadas em consideração e objeto de produção do cuidado. Para tanto, faz-se necessário considerar a vida do outro como importante, respeitando a sua autonomia e atendendo às suas necessidades de saúde numa perspectiva de integralidade e complexidade. ${ }^{(16)}$

A fim de atender tais necessidades, no cenário das políticas públicas brasileira, o Decreto Presidencial $n^{\circ} 7508 / 11$, que regulamenta a Lei Orgânica do Sistema Único de 
Saúde (Lei nº 8080/90), reconhece oficialmente a atenção básica como porta de entrada dos serviços, trazendo melhorias relacionadas a resolutividade baseadas nas necessidades da população, qualificando o acesso e tornando o cuidado eficiente (17). A ESF e os CAPS são considerados serviços que não necessitam de encaminhamentos e que devem assegurar a integralidade da atenção independentemente do nível de complexidade do serviço.

Como base nesses princípios, observa-se que o itinerário dos usuários não conduz a uma produção do cuidado que respeite os princípios da universalidade, equidade e integralidade.

Na primeira categoria, que versa sobre o acesso aos serviços da RAPS já demonstra uma dificuldade na produção do cuidado que passe pelas dificuldades no acesso ao serviço de saúde, condição que se exacerba nos serviços não especializados como a ESF. Estudo realizado sobre as práticas da ESF enquanto porta de entrada e acesso dos usuários ao serviço especializado mostra que, apesar de promover ampliação da assistência ao nível básico, a estratégia não assegura um acesso complexo e integral, interrompendo a continuidade da assistência e a ligação entre os diferentes serviços. ${ }^{(18)}$

Assim como apresentado nos resultados do presente estudo realizado diretamente com profissionais da equipe de enfermagem, outro estudo realizado com gestores (19) evidencia a partir de um outro olhar, que a equipe de enfermagem, ao realizar o encaminhamento do usuário, compreende tal ação como forma de ter assegurado o acesso à RAPS e que o seu papel enquanto parte da rede foi cumprido. No entanto, o cuidado não alcança o seu objetivo da integralidade, tornando-se fragmentado. Logo, entende-se pela necessidade de uma melhor articulação entre a ESF e os serviços especializados de atenção psicossocial, buscando uma nova conformação para os CAPS e sua integração participativa com os serviços da atenção básica.

Estudo realizado sobre a experiência do uso do Crack e sua interlocução com a clínica, reafirma a dificuldade que os usuários têm de acessar os serviços, impedindo que eles tenham suas necessidades atendidas. ${ }^{(3)}$ A prática da equipe de saúde ainda é pautada na lógica da produtividade, direcionada pela realização de procedimentos e caracteriza o acesso a partir de ações programadas para uma população específica ou situações de crise do usuário, configurando-se numa prática produtivista na qual não são observados os princípios da integralidade e complexidade inerentes ao cuidado.

Muitas vezes são desconsiderados os determinantes sociais, os quais refletem grande vulnerabilidade na vida das pessoas que se tornam usuárias de álcool e outras drogas. Estudo realizado com estudantes de uma Universidade Pública na Colômbia concluiu que dos seus participantes, na sua grande maioria, apresentavam iniquidades social, os tornando vulneráveis e expostos as situações estressantes, sendo um ponto de partida para o uso de substâncias como álcool e outras drogas. ${ }^{(19)}$

Daí a importância de políticas públicas que leve em consideração os determinantes sociais para as discussões acerca da produção do cuidado aos usuários dos serviços de atenção psicossocial. No Brasil, a Atenção Primária à Saúde é responsável por realizar acompanhamento dos casos e ações de redução de danos nos territórios. No entanto, ainda há uma fragilidade no processo de cuidar quando os trabalhadores de saúde afirmam que não sabem o que fazer com estes usuários, e sua intervenção se limita a encaminhá-los para o CAPS-AD. ${ }^{(3)}$ Tal desconhecimento também é evidenciado na compreensão dos participantes deste estudo.

A segunda categoria do estudo trata do acolhimento aos usuários na RAPS, nesse ponto os resultados trazem que apesar de os profissionais da equipe de enfermagem não saberem como conduzir o acompanhamento do usuário e de centrar suas ações no ato de 
encaminhar, observou-se nesta pesquisa que há uma preocupação por parte dessa equipe de não deixar o usuário sair do serviço sem um momento de escuta, o que nos permite inferir que na perspectiva do acolhimento, há uma tentativa de produzir o cuidado.

Estudos ${ }^{(16)}$ reconhecem que a produção do cuidado por meio do acolhimento é importante e diz respeito a forma como o usuário é abordado, considerando seu papel social como cidadão de direitos e deveres, e tratá-lo como protagonista do seu processo de cuidado e não como um ser passivo.

$\mathrm{O}$ ato de escutar é uma maneira de contribuir com a produção do cuidado, a partir de um olhar diferenciado que cada um traz e que pode cooperar para a compreensão e comunicação entre usuários e trabalhadores da saúde. Assim, tem-se a possibilidade de que o usuário ocupe um lugar de agente ativo na produção de sua saúde. (20)

Ainda sobre acolhimento, uma pesquisa ${ }^{(18)}$ que avaliou a forma como é conduzido o acolhimento em serviços especializados, possibilitou verificar uma prática pautada numa postura ética e humanística, em prol de uma relação de confiança, escuta e compromisso para com os usuários, seus familiares e demais profissionais de saúde, guiada pela cultura da solidariedade e legitimação dos princípios estruturantes do SUS.

No entanto vale destacar que os resultados do presente estudo evidenciam que os participantes atuantes nos CAPS utilizam o acolhimento por este ser uma ferramenta que produz um cuidado integral e resolutivo, entretanto aqueles que atuam na ESF entendem que apesar da importância do acolhimento, os usuários devem ser encaminhados para os serviços especializados demonstrando desta forma que há falta de qualificação para realizarem o cuidado extramuros das unidades especializadas. Desta forma, vale considerar a necessidade de qualificação dos profissionais da ESF com conhecimentos sobre a atenção psicossocial, para que estes consigam dar resolutividade às necessidades de saúde dos usuários de álcool e outras drogas.

Importante reforçar que o acesso transversaliza as práticas de cuidado à saúde, num itinerário que vai desde a "porta de entrada" aos distintos espaços de cuidados inerentes a RAPS. Desse modo, os profissionais que atuam na Rede não devem centrar a sua atuação no ato de receber o usuário e encaminhá-lo para outro serviço, mas buscar escutar, orientar e planejar uma assistência que atenda às reais necessidades desse em qualquer serviço da RAPS. ${ }^{(2)}$

Esse cenário deve prevalecer em toda a rede e não em pontos específicos, como foi evidenciando nos resultados que quando se trata de uma unidade especializada como o CAPS $\mathrm{AD}$, fica clara a produção de cuidado com acolhimento e escuta ativa, dando conta das necessidades e tentando atender as demandas que os usuários trazem, embora em alguns momentos tenham que recorrer aos dispositivos da atenção secundária, como o hospital.

O cuidado compartilhado entre a ESF e os CAPS ainda representa um desafio. Estudo (16) que buscou analisar o vínculo-responsabilização das equipes da ESF, aponta que o vínculo não se estabelece, muitas vezes, pela falta de interesse e apropriação teórica das equipes em discutir os casos que demandam o cuidado de saúde mental no território.

Estudo de revisão sistemática ${ }^{(21)}$ indica que a ESF vai além de ser a porta de entrada na RAPS, mas representa o primeiro contato do usuário com o sistema de saúde, por se configurar como o ambiente mais adequado para instituir medidas educativas e preventivas.

Assim, podemos destacara como estratégia de intervenção, a utilização de técnicas terapêuticas sucintas e de pequena duração, a exemplo da "Intervenção Breve", uma técnica terapêutica direcionada a mudança de conduta, na busca por redução de danos, ajudando os usuários a perceber que tal uso lhes causam riscos, tornando-se uma motivação para que reduzam ou deixem de consumir tais substâncias. ${ }^{(22)}$ 
Desse modo, é imprescindível garantir estratégias para o acolhimento aos usuários e que estes se sintam confiantes e estabeleçam vínculos com os profissionais de saúde e os serviços, de modo a viabilizar um cuidado que vá ao encontro das reais necessidades de usuários e famílias. Como outras estratégias destacamos o acolhimento, o PTS, a referência técnica, a visita domiciliar e as reuniões de equipe como importantes ferramentas para a produção do cuidado aos usuários, práticas que requer um comprometimento por parte de toda equipe. ${ }^{(23)}$

Destacamos que os resultados trazem como modalidades de cuidados oferecidos nos CAPS, as assembleias, como recurso terapêutico produtor de autonomia, na busca pela maior participação e responsabilização dos usuários em seu tratamento, além de representar um importante instrumento institucional. Entretanto nesse estudo alguns profissionais relataram que conhecem esse tipo de ferramenta, porém não o colocam em prática, negligenciando uma estratégia de cuidado que pode ser utilizada nos serviços.

Estudo ${ }^{(24)}$ de reflexão teórica sobre intervenções terapêuticas utilizando-se das assembleias como dispositivos grupais e, marcante na constituição das políticas públicas brasileiras e dos CAPS, demonstrou que com a utilização desse dispositivo, os usuários passaram a enfrentar melhor seus limites que são impostos pela doença. Portanto, ao enfatizar os aspectos saudáveis nas exigências do cotidiano, a doença assume um papel secundário, dando lugar a experiência de empoderamento, o que por si só, pode ser terapêutico.

O uso das assembleias, pode vir a ser uma estratégia para a construção de vínculos, considerando que os profissionais da equipe de enfermagem têm o entendimento sobre o conceito de vínculo e buscam exercê-lo, todavia ainda lhes faltam a compreensão quanto à necessidade de desenvolver estratégias para construção de vínculo.

A terceira categoria, trata do vínculo entre a equipe de enfermagem e os usuários da RAPS, sendo uma dificuldade na produção do cuidado e, os profissionais atuantes nos serviços especializados trazem que não há uma articulação entre a ESF e o CAPS para construção de um cuidado compartilhado e integral, uma vez que os profissionais dos CAPS apontam a falta de interesse e apropriação teórica da equipe de ESF para discutir os casos que demandam o cuidado de saúde mental na APS, o que favorece a uma atitude de desresponsabilização e não geração de vínculo entre usuários e equipe. Condição que também foi observada em dois outros estudos com profissionais da enfermagem sobre a produção do cuidado. ${ }^{(25-26)}$

Desta forma, para que ocorra o vínculo, é importante contar com profissionais capacitados para lidar com os usuários de álcool e outras drogas em toda a RAPS, não apenas nos serviços especializados. Assumindo a necessidade e relevância de uma atuação com base no profissional de referência, o que não foi percebido no cenário estudado, seja por não compreenderem a sua relevância ou por um entendimento equivocado de que este papel é apenas do enfermeiro. O técnico de referência deve ser aquele que tem como responsabilidade o monitoramento do usuário a partir de um PTS. ${ }^{(3)}$

Pesquisa ${ }^{(26)}$ desenvolvida numa cidade do Nordeste brasileiro e discutiu como os sujeitos sociais compreender as práticas de gerenciamento do cuidado da enfermeira na ESF, dentre elas o dispositivo relacionado ao vínculo, evidenciou a importância de uma atuação com base num profissional de referência dentro dos serviços da RAPS e pautando-se no matriciamento das equipes quer seja no nível básico ou especializado. A principal função do técnico de referência pauta-se na elaboração e aplicação prática do PTS, o qual implica num diagnóstico situacional, na aproximação entre usuário, família e profissionais resultando em práticas terapêuticas individuais ou coletivas e na geração de vínculo entre equipe e usuários. 
Devendo ser uma prática não apenas dos profissionais atuantes no CAPS, mas para todos da rede.

Diante desse cenário, a produção do cuidado pela equipe de enfermagem junto ao usuário e seus familiares, pode ser conduzida em equipe multiprofissional, que possibilita ampliar o diálogo entre os diferentes atores sociais, potencializando vínculos, além de estabelecer responsabilidades entre os serviços de saúde e a rede de relações do usuário.

Desse modo, o PTS, precisa ser construído junto com o usuário como estratégia de corresponsabilização e estímulo à sua autonomia, com vistas ao resgate da cidadania, autoestima e poder contratual, tendo como pilar o projeto de vida de cada usuário.

Assim como foi evidenciado nos resultados deste estudo, um outro ${ }^{(27)}$ demonstrou que profissionais da ESF também desconhecem o PTS. Um dispositivo que precisa ser disseminados para além da rede de atenção psicossocial, considerando sua relevância no cuidado oferecido pela ESF, por possibilitar auxilia na gestão do cuidado às pessoas, valorizar a singularidade destes no momento em que considera seu modo de ser, pensar e agir, e, demonstrar um cenário no qual os profissionais podem e devem desenvolver um cuidado interdisciplinar.

Uma revisão sistemática desenvolvida por estudiosos da área, na Austrália, buscou sintetizar as evidências quanto as estratégias para um cuidado integral aos usuários de álcool e outras drogas e pôde concluir que apesar de os trabalhadores da saúde reconhecer os benefícios de um cuidado integral, ainda são incipientes as estratégias utilizadas para produzir um cuidado biopsicosocioespiritual no contexto de atendimento ao usuário de álcool e outras drogas. ${ }^{(28)}$

\section{Conclusão}

O estudo evidenciou que a produção do cuidado profissional prestado pela equipe de enfermagem aos usuários de álcool e outras drogas na RAPS investigada dar-se por meio do acesso, acolhimento e vínculo, no entanto apenas nas unidades especializadas e não em toda a rede.

As práticas de cuidado precisam ser mais bem articuladas entre os distintos cenários da RAPS para que de fato seja produzido um cuidado efetivo e de qualidade para os usuários e respectivas famílias, na rede como um todo e não apenas nas unidades especializadas. É preciso reconhecer a ESF como a porta de entrada, na qual toda equipe precisa ser preparada para cuidar dos usuários frente as suas necessidades de modo a reconhecer suas particularidades e vulnerabilidade diante das iniquidades e determinantes sociais.

Assume-se como limitação desse estudo, a participação de apenas um grupo de profissionais, aqueles do campo da Enfermagem, entendendo que com a participação interdisciplinar, seja possível uma maior discussão a partir de diferentes olhares.

Espera-se que as discussões vislumbradas nesta pesquisa sejam aprofundadas em novos estudos, acerca das práticas de cuidados ofertadas por profissionais da equipe de enfermagem aos usuários de álcool e outras drogas, e ampliem as possibilidades de construir novas práticas e refletir sobre aquelas que vem sendo desenvolvidas. 


\section{Referências bibliográficas}

1. Brasil. Ministério da Saúde. A Política para Atenção Integral a Usuários de Álcool e outras Drogas. Brasília: Ministério da Saúde; 2003.

2. Bastos FI, Vasconcellos MT, Boni RB, Reis NB, Coutinho CF. (Org). III Levantamento Nacional sobre o uso de drogas pela população brasileira. Rio de Janeiro: Fiocruz/Icict; 2017. Disponível em: https://www.arca.fiocruz.br/handle/icict/34614

3. Souza MC, Afonso ML. Saberes e práticas de enfermeiros na saúde mental: desafios diante da reforma psiquiátrica. Rev. Interinst. Psicol. 2015;8(2):332-347. Disponível em: http://pepsic.bvsalud.org/pdf/gerais/v8n2/v8n2a04.pdf

4. Ortiz SE, Domingues EA, Prado KL, Barbosa PL. Prática de enfermagem o centro de atenção psicossocial. Rev. Bras. Enferm. 2020;73(1). Disponível em: DOI: 10.1590/0034-7167-2018-0175

5. Portaria $n^{\circ} 3.088$ de 23 de dezembro de 2011. Institui a Rede de Atenção Psicossocial para pessoas com sofrimento ou transtorno mental e com necessidades decorrentes do uso de crack, álcool e outras drogas, no âmbito do Sistema Único de Saúde (SUS). Diário Oficial da União. Brasília; 2011. Disponível em: http://bvsms.saude.gov.br/bvs/saudelegis/gm/2011/prt3088_23_12_2011_rep.html.

6. Fernandes HN, Eslabão AD, Mauch LM, Franchini B, Coimbra VC. A práxis do cuidado em saúde mental na atenção ao uso e abuso de álcool. Cienc cuid. saúde. 2012;11(4):827-831. DOI: 10.4025/cienccuidsaude.v11i4.15407

7. Sousa FD, Sousa AL. Uma revisão sobre as redes de atenção à saúde no Brasil. Revista Científica Multidisciplinar Núcleo do Conhecimento. 2018;1(11):57-75. DOI: 10.32749/nucleodoconhecimento.com.br/saude/saude-no-brasil

8. Terezinha ZM, Fontoura CM. Bradt, FM. Ziegler HM, Godinho, BK. Percepção dos trabalhadores de saúde sobre o cuidado as pessoas com crise a rede de atenção psicossocial. Esc. Ana Nery. 2016;20(3):e20160059. DOI: 10.5935/14148145.20160059

9. Varela DS, Sales IM, Dantas FM, Monteiro CF. Rede de saúde no atendimento ao usuário de álcool, crack e outras drogas. Esc Ana Nery. 2016;20(2):296-302. DOI: $10.5935 / 1414-8145.20160040$

10. Franco TE, Merhy EE. Trabalho, produção do cuidado e subjetividade em saúde. São Paulo: Hucitec; 2013.

11. Feuerwerker LCM. Micropolítica e saúde: produção do cuidado, gestão e formação. Porto Alegre: Rede UNIDA; 2014.

12. Minayo, MC. O desafio do conhecimento: pesquisa qualitativa em Saúde. 12. ed. São Paulo: Hucitec; 2014. 
13. Tong A, Sainsbury P, Craig J. Consolidated criteria for reporting qualitative research (COREQ): a 32- item checklist for interviews and focus group. Int J Qual Heal Care. 2007;19(6):349-57. Disponível https://academic.oup.com/intqhc/article/19/6/349/1791966

14. Bardin L. Análise de conteúdo. São Paulo: Edições 70; 2016.

15. Amorim RG, Lavrador MCC. A Perspectiva da Produção de Cuidado pelos Trabalhadores de Saúde Mental. Psicologia Ciência e Profissão. 2017;37(2):273-288. Disponível em: https://www.scielo.br/pdf/pcp/v37n2/1982-3703-pcp-37-2-0273.pdf

16. Subrinho LQ, Sena EL, Santos VT, Carvalho PA. Cuidado ao consumidor de drogas: percepção de enfermeiros da estratégia de saúde da família. Saúde Soc. 2018;27(3):834844. DOI: $10.1590 / \mathrm{s} 0104-12902018180079$

17. Decreto $n^{\circ} 7.508$ de 28/2011. Dispõe sobre a organização do Sistema Único de Saúde, o planejamento da saúde, a assistência à saúde e a articulação Inter federativa e dá outras providências [Internet] Diário Oficial da União. Brasília; 2011. Disponível em: http://www.planalto.gov.br/ccivil_03/_ato2011-2014/2011/decreto/d7508.htm

18. Souza AC, Amarante PD, Abrahão AL. Inclusão da saúde mental na atenção básica à saúde: estratégia de cuidado no território. Rev Bras Enferm. 2019;72(6):1677-1683. DOI: 10.1590/0034-7167-2018-0806

19. Narváez NEB, Rivero MO, Guerra EH. Social determinants in the consumption of psychoactive substances in university students in 2016. Enfermería (Montevideo). 2018;7(2):12-35. Disponível em: http://www.scielo.edu.uy/pdf/ech/v7n2/en_23936606-ech-7-02-12.pdf

20. Agonigi RC, Carvalho SM, Freire MAM, Gonçalves LF. The production of care in the routine of Family Health Teams. Rev. Bras. Enferm. 2018;71(Suppl 6):2659-2665. Disponível em: https://www.scielo.br/pdf/reben/v71s6/0034-7167-reben-71-s62659.pdf

21. Pereira MO, Anginoni BM, Ferreira NC, Oliveira MAF, Vargas D, Colvero LA. Efetividade da intervenção breve para o uso abusivo de álcool na atenção primária: revisão sistemática. Rev. Bras. Enferm. 2013; 66(3):420-428. Disponível em: https://www.scielo.br/pdf/reben/v66n3/a18v66n3.pdf

22. Abreu AMM, Jomar RT, Taets GGC, Souza MHN, Fernandes DB. Screening and Brief Intervention for the use of alcohol and other drugs. Rev. Bras. Enferm. 2018;71(Suppl 5):2258-2263. Disponível em: https://www.scielo.br/pdf/reben/v71s5/0034-7167reben-71-s5-2258.pdf

23. Amorim AC, Assis MM, Santos AM. Vínculo e responsabilização como dispositivos para produção do cuidado na estratégia de saúde da família. Rev. baiana saúde pública. 2014;38(3):539-554. DOI: 10.5327/Z0100-0233-2014380300004 
24. Junqueira AM, Carniel IC, Montovani A. As assembleias como possibilidade de cuidado em saúde mental em um CAPS. Rev. Nesme 2015;12(1):31-40. Disponível em: http://pepsic.bvsalud.org/pdf/vinculo/v12n1/v12n1a06.pdf

25. Ferreira TP, Sampaio J, Souza AC, Oliveira DL, Gomes LB. Produção do cuidado em Saúde Mental: desafios para além dos muros institucionais. Interface. 2017;21(61):37384. DOI: 10.1590/1807-57622016.0139.

26. Silva SS, Assis MM, Santos AM. Enfermeira como protagonista do gerenciamento do cuidado na estratégia saúde da família: diferentes olhares analisadores. Texto Contexto Enferm. 2017;26(3):e1090016. DOI: 10.1590/0104-07072017001090016.

27. Silva AI, Loccioni MF, Orlandini RF, Rodrigues J, Peres GM, Maftum MA. Projeto Terapêutico Singular para profissionais da estratégia de saúde da família. Cogitare Enferm. 2016;21(3):01-08. DOI: 10.5380/ce.v21i3.45437

28. Savic M, Best D, Manning V, Lubman DI. Strategies to facilitate integrated care for people with alcohol and other drug problems: a systematic review. Subst Abuse Treat Prev Policy. 2017;12(19):1-12. DOI: 10.1186/s13011-017-0104-7

Participação dos autores: a) Planejamento e concepção do trabalho; b) Coleta de dados; c) Análise e interpretação de dados; d) Redação do manuscrito; e) Revisão crítica do manuscrito.

P. M. C. d. B. contribuiu em a, b, c, d, e; M. B. A. em c, d, e; V. M. O. em d, e; M. d. P. S. d. S. N. em d, e; S. C. O. P. em d, e; R. S. D. S. em c, d, e.

Editora científica responsável: Dra. Natalie Figueredo 